\title{
Reconstruction plates used in the surgery for mandibular discontinuity defect
}

\author{
Guk-Jin Seol, Eun-Gyu Jeon, Jong-Sung Lee, So-Young Choi, Jin-Wook Kim, Tae-Geon Kwon, Jun-Young Paeng \\ Department of Oral and Maxillofacial Surgery, Kyungpook National University School of Dentistry, Daegu, Korea
}

\begin{abstract}
J Korean Assoc Oral Maxillofac Surg 2014;40:266-271)
Objectives: The purpose of this study was to analyze the survival rate of reconstruction plates that were used to correct mandibular discontinuity defects.

Materials and Methods: We analyzed clinical and radiological data of 36 patients. Only discontinuous mandibular defect cases were included in the study. Reconstruction plate survival rate was analyzed according to age, gender, location of defect, defect size, and whether the patient underwent a bone graft procedure, coronoidectomy, and/or postoperative radiation therapy (RT).

Results: Plate-related complications developed in 8 patients, 7 of which underwent plate removal. No significant differences were found in plate survival rate according to age, gender, location of defect, defect size, or whether a bone graft procedure was performed. However, there were differences in the plate survival rate that depended on whether the patient underwent coronoidectomy or postoperative RT. In the early stages ( $9.25 \pm 5.10$ months), plate fracture was the most common complication, but in the later stages ( $35.75 \pm 17.00$ months), screw loosening was the most common complication. Conclusion: It is important to establish the time-related risk of complications such as plate fracture or screw loosening. Coronoidectomy should be considered in most cases to prevent complications. Postoperative RT can affect the survival rate and hazard rate after a reconstruction plate is fitted.
\end{abstract}

Key words: Mandibular reconstruciton, Complications, Survival rates

[paper submitted 2014. 8. 12 / revised 2014. 9. 16 / accepted 2014. 9. 29]

\section{Introduction}

Surgical treatment for malignant tumors, benign tumors, trauma, or jaw necrosis often requires subsequent treatment to recover functionality and correct aesthetic problems. Mandibular reconstruction plates are used in oral and maxillofacial surgery for mandibular defect reconstruction. Because the mandible plays a central role in function and aesthetics, the loss of jaw continuity can severely impair a patient's jaw integrity, and severely affect the patient's self-perception and self-confidence ${ }^{1}$.

The main goals of mandibular reconstruction are to restore the continuity of the mandible arch and provide support to the outer soft tissue, which in turn can support functional and aesthetic rehabilitation and improved postoperative quality of life.

\section{Jun-Young Paeng}

Department of Oral and Maxillofacial Surgery, Kyungpook National University School of Dentistry, 2175 Dalgubeol-daero, Jung-gu, Daegu 700-705, Korea TEL: +82-53-600-7551 FAX: +82-53-426-5365

E-mail: jypaeng@gmail.com

(c) This is an open-access article distributed under the terms of the Creative Commons Attribution Non-Commercial License (http://creativecommons.org/licenses/by-nc/3.0/), which permits unrestricted non-commercial use, distribution, and reproduction in any medium, provided the original work is properly cited.

Copyright (C) 2014 The Korean Association of Oral and Maxillofacial Surgeons. All rights reserved.
The first generation of clinically available reconstruction plates was introduced in the mid-1970s ${ }^{2}$. Since the early 1980s, the standard treatment for a mandibular discontinuity defect has been the use of a rigid reconstruction plate with concurrent or subsequent osseous reconstruction ${ }^{3}$. Recently, the effectiveness of osteosynthesis has improved with the use of titanium reconstruction plates, which provide better biocompatibility, and locking screws for biomechanical improvement. With these improvements, modern reconstruction plates can provide excellent anchorage and rigid fixation ${ }^{4,5}$. However, postoperative plate-related complications (Fig. 1), including plate exposure, plate fracture, screw loosening, and infection still occur occasionally ${ }^{6,7}$.

Therefore, this study aimed to evaluate the factors associated with postoperative complications and analyze the survival rate of the mandibular reconstruction plate.

\section{Materials and Methods}

In this study, 36 patients (20 male and 16 female) who had discontinuity defects were included among a total of 77 patients who underwent reconstruction plate procedures. Only cases of discontinuous mandibular defect were included in 

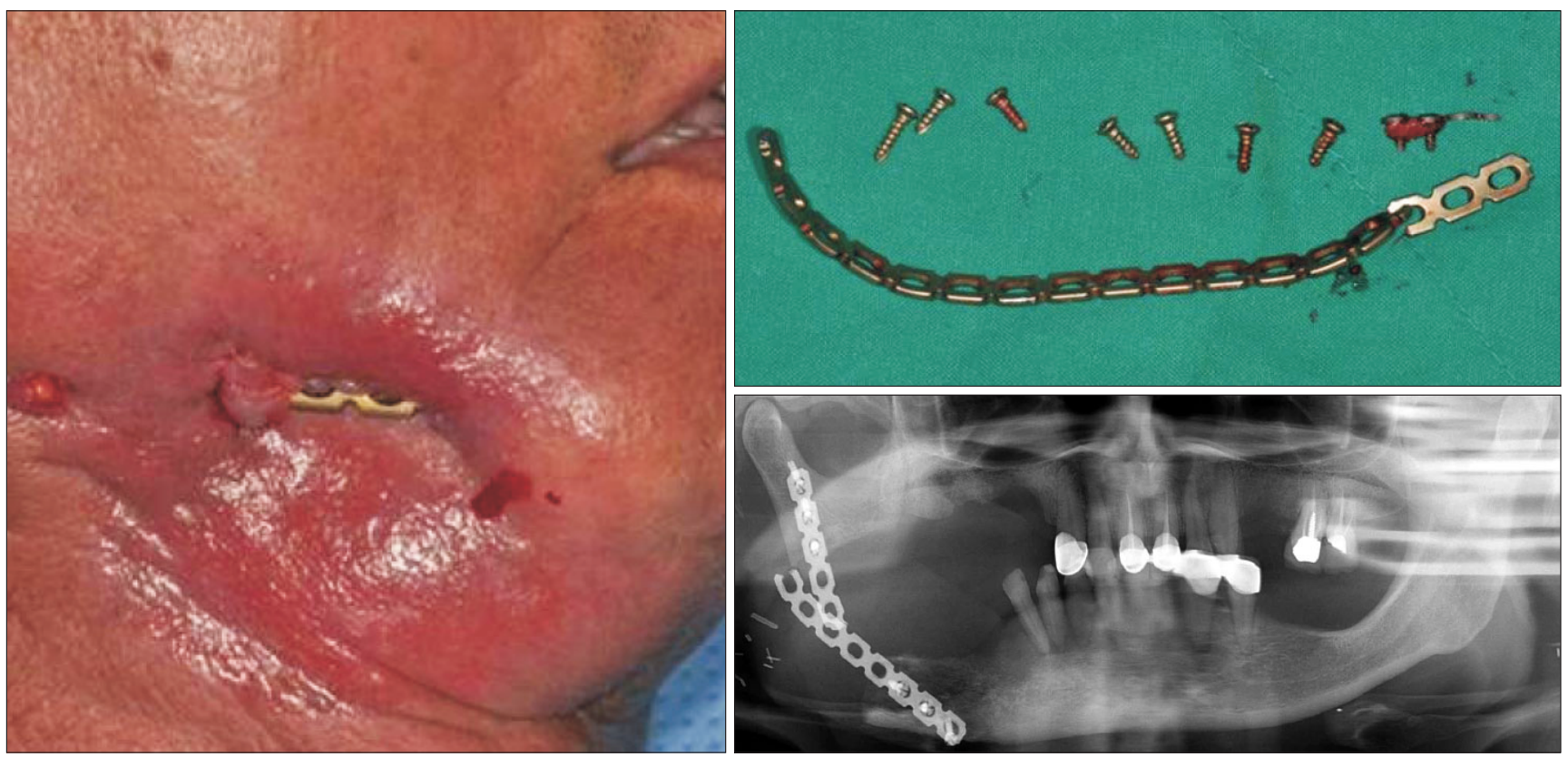

Fig. 1. Plate-related complications: plate exposure, plate fracture, and screw loosening. Guk-Jin Seol et al: Reconstruction plates used in the surgery for mandibular discontinuity defect. J Korean Assoc Oral Maxillofac Surg 2014

the study; the other 41 patients with mandibular continuity defects were excluded. The patients visited the Kyungpook National University Department of Oral and Maxillofacial Surgery between 2004 and 2013. They underwent a surgical operation and reconstruction plates were used to correct the mandibular discontinuity defect and to reconstruct the defect. The surgical procedures were performed while the patients were under general anesthesia. The clinical history, radiological data, and survival rate of the reconstruction plates were analyzed according to age, gender, location of defect, removal of the coronoid process, use of bone graft in the reconstruction, and whether postoperative radiation therapy (RT) was performed. Synthes Reconstruction Plates (Synthes, Westchester, PA, USA) or Jeil Medical Maxi Plates 2.4 (Jeil Medical, Seoul, Korea) were used as the reconstruction plates. Twenty-seven Synthes Reconstruction Plates and nine Jeil medical Maxi Plates were used for the mandibular reconstruction. The survival rate of the reconstruction plate according to gender, whether a bone graft procedure and/or coronoidectomy were performed, the overall survival rate, and the hazard rate were analyzed for statistical significance by Fisher's exact test and Pearson's chi-squared test, and confirmed with a log-rank test. All tests were performed with the R (R Development Core Team, 2013; http://www. r-project.org) software package on a personal computer, and $P<0.05$ was accepted as the level of statistical significance. This study was approved by the institutional review board of
Table 1. Disease processes underlying mandibular discontinuity defect cases

\begin{tabular}{lcc}
\hline Pathological diagnosis & Cases $(\mathrm{n})$ & Frequency $(\%)$ \\
\hline Malignant tumor & 15 & 41.7 \\
Benign tumor & 12 & 33.4 \\
Fracture & 7 & 19.4 \\
Osteomyelitis & 2 & 5.6 \\
\hline
\end{tabular}

Guk-Jin Seol et al: Reconstruction plates used in the surgery for mandibular discontinuity defect. J Korean Assoc Oral Maxillofac Surg 2014

Kyungpook National University Hospital (No. 2014-07-016).

\section{Results}

The mean age of the patients was $45.7 \pm 17.0$ years and the mean follow-up period was 23.9 \pm 20.0 months (median 19 months) and ranged from 1 week to 7 years and 6 months. The patients had a number of different diagnoses, including malignant tumor presence, benign tumor, severe mandibular fracture, and large cystic lesions. Malignant tumors accounted for 15 cases $(41.7 \%)$, benign tumors for 12 cases $(33.4 \%)$, fractures for 7 cases (19.4\%), and osteomyelitis for 2 cases (5.6\%). (Table 1) The overall postoperative plate-related complications and affecting factors are summarized in Table 2. Postoperative plate-related complications occurred in 8 (4 plate fracture and 4 screw loosening cases) of 36 patients (22.2\%); of these 8 , 7 patients (87.5\% of patients with complications) underwent plate removal. One patient experienced a screw loosening 
Table 2. Overall postoperative plate-related complications

\begin{tabular}{|c|c|c|c|c|c|c|c|c|}
\hline \multirow{2}{*}{ Variable } & \multicolumn{8}{|c|}{ Assessment } \\
\hline & 1 & 2 & 3 & 4 & 5 & 6 & 7 & 8 \\
\hline Age (yr) & 42 & 70 & 57 & 23 & 40 & 69 & 48 & 48 \\
\hline Gender & Male & Male & Male & Male & Female & Male & Female & Male \\
\hline Diagnosis & MT & MT & MT & BT & BT & MT & MT & BT \\
\hline LOD & Right angle & Right angle & Left angle & Left angle & Right body & Left angle & Right angle & Right angle \\
\hline Bone graft & 0 & $\times$ & 0 & 0 & 0 & $\times$ & $\times$ & $\times$ \\
\hline Plate type & Synthes $^{1}$ & Synthes ${ }^{1}$ & Synthes ${ }^{1}$ & $\mathrm{Jeil}^{2}$ & $\mathrm{Jeil}^{2}$ & $\mathrm{Jeil}^{2}$ & Synthes $^{1}$ & Synthes $^{1}$ \\
\hline Coronoidectomy & $\times$ & $\times$ & $\times$ & $\times$ & $\times$ & $\times$ & $\times$ & $\times$ \\
\hline Postoperative RT & O & 0 & $\times$ & $\times$ & $\times$ & $\times$ & $\mathrm{O}$ & $\times$ \\
\hline Complications & Loosen & Fracture & Loosen & Fracture & Loosen & Loosen & Fracture & Fracture \\
\hline Plate removal & O & 0 & $\times$ & $\mathrm{O}$ & O & O & O & $\mathrm{O}$ \\
\hline F/U duration (m) & 36 & 14 & 14 & 71 & 54 & 40 & 10 & 2 \\
\hline
\end{tabular}

(MT: malignant tumor, BT: benign tumor, LOD: location of defect, RT: radiation therapy, F/U: follow-up)

${ }^{1}$ Synthes Reconstruction Plates, ${ }^{2}$ Jeil Medical Maxi Plates.

Guk-Jin Seol et al: Reconstruction plates used in the surgery for mandibular discontinuity defect. J Korean Assoc Oral Maxillofac Surg 2014

A

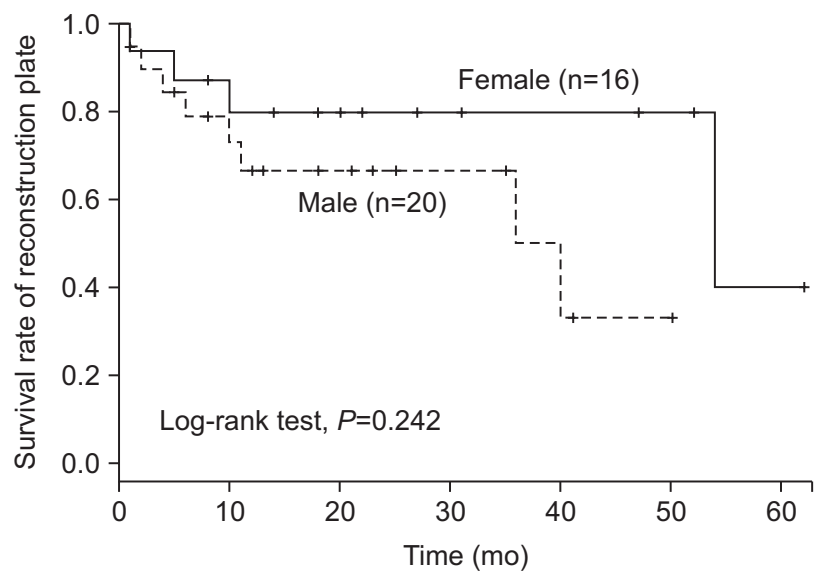

B

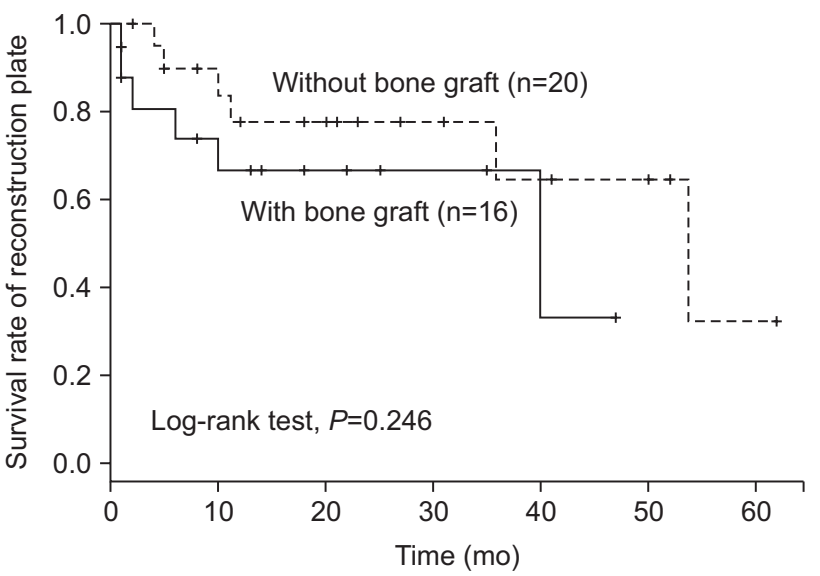

Fig. 2. A. No significant difference was found for the reconstruction plate survival rate between males and females. B. No significant difference was found for the reconstruction plate survival rate between patients that did or did not receive a bone graft.

Guk-Jin Seol et al: Reconstruction plates used in the surgery for mandibular discontinuity defect. J Korean Assoc Oral Maxillofac Surg 2014

complication but refused the removal surgery.

There was no relationship between the survival rate of the reconstruction plate and the gender of the patient or the location of the defect.(Fig. 2. A) Plate fracture occurred in $4.8 \%$ of the patients $(n=1)$ who received a bone graft and in $20 \%$ of patients $(n=3)$ who did not receive a bone graft. However, the difference between the two groups was not statistically significant (log-rank test, $P=0.246$ ).(Fig. 2. B)

The survival rate of the reconstruction plate was significantly higher in patients who underwent coronoidectomy than in those who did not (log-rank test, $P=0.032$ ).(Fig. 3) None of the 9 patients that underwent mandibular reconstructive surgery with coronoidectomy showed any complications; in contrast, 7 out of 27 patients $(25.9 \%)$ that did not undergo coronoidectomy had their reconstruction plates removed.(Fig. 4)

The main reasons for plate removal were fracture or recon-

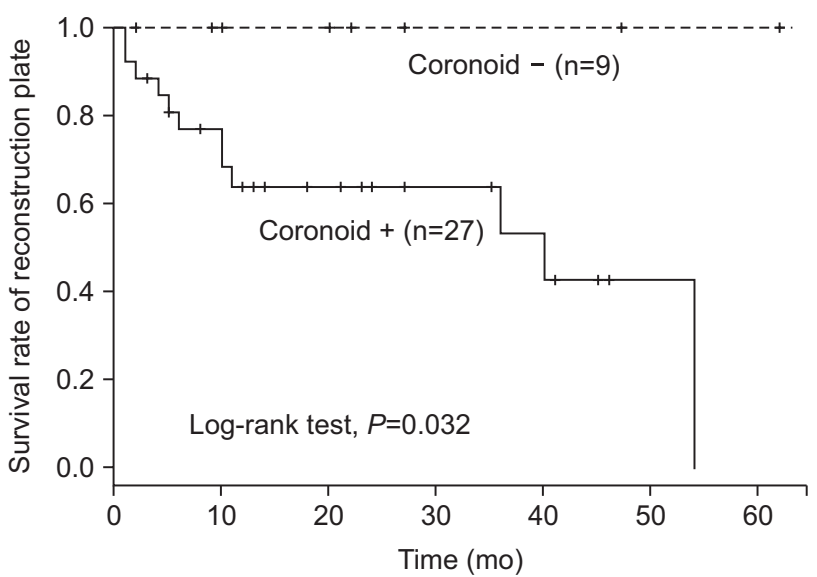

Fig. 3. A significant difference was found between patients who received a coronoidectomy and those that did not.

Guk-Jin Seol et al: Reconstruction plates used in the surgery for mandibular discontinuity defect. J Korean Assoc Oral Maxillofac Surg 2014 

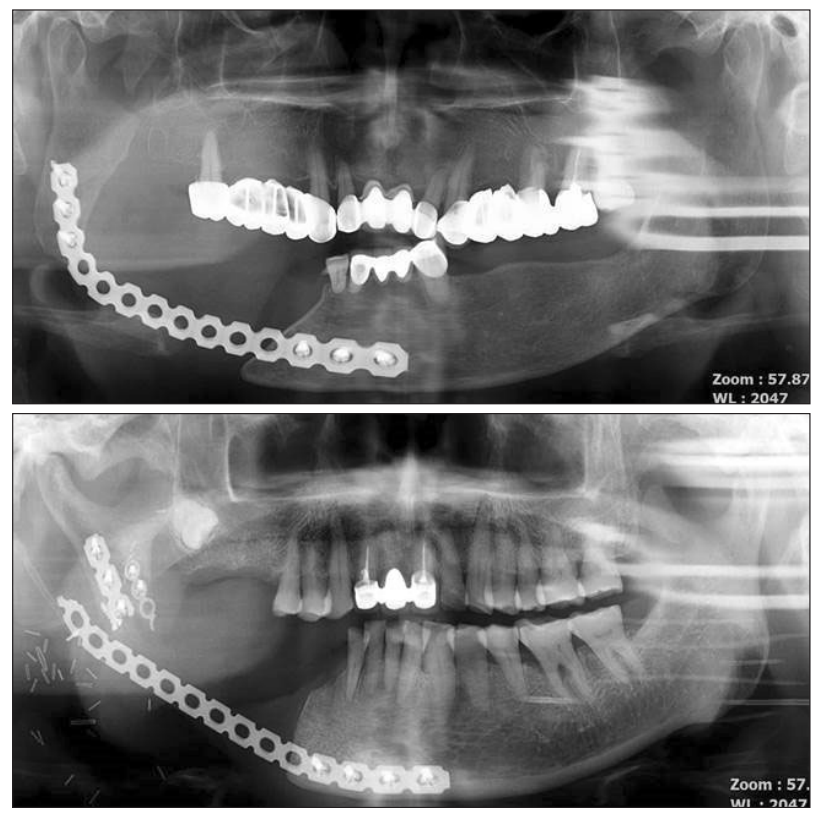

Fig. 4. In two similar cases, reconstruction plate fracture did not occur in the patient who underwent coronoidectomy, while plate fracture occurred in the patient who did not undergo coronoidectomy. Guk-Jin Seol et al: Reconstruction plates used in the surgery for mandibular discontinuity defect. J Korean Assoc Oral Maxillofac Surg 2014

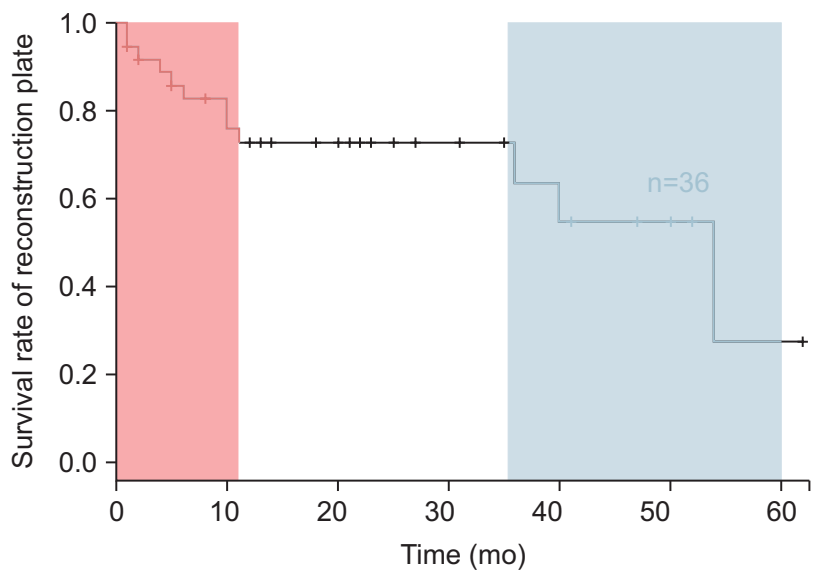

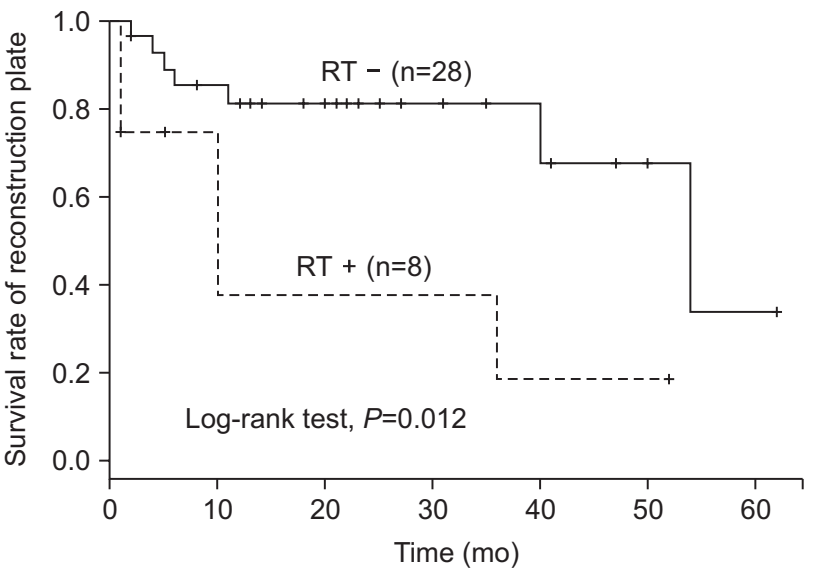

Fig. 7. Survival curves showing the decreased survival rate of reconstruction plates following postoperative radiation therapy (RT). Guk-Jin Seol et al: Reconstruction plates used in the surgery for mandibular discontinuity defect. J Korean Assoc Oral Maxillofac Surg 2014

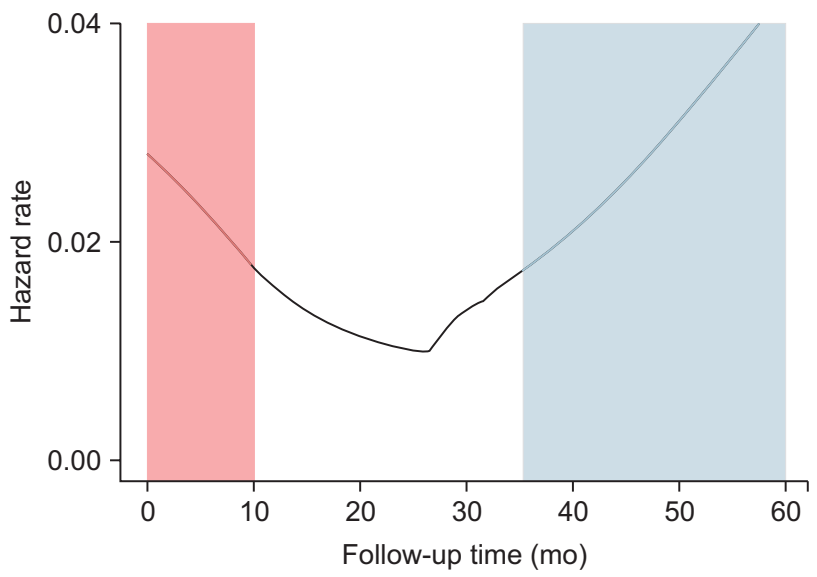

Fig. 5. The overall survival rate, the survival curve over the first 10 months and after 35 months. A similar trend was apparent for the hazard rate, the risk of complications was low from the 10 to 35 months. Red box: high risk of plate fracture. Blue box: high risk of screw loosening. Guk-Jin Seol et al: Reconstruction plates used in the surgery for mandibular discontinuity defect. J Korean Assoc Oral Maxillofac Surg 2014
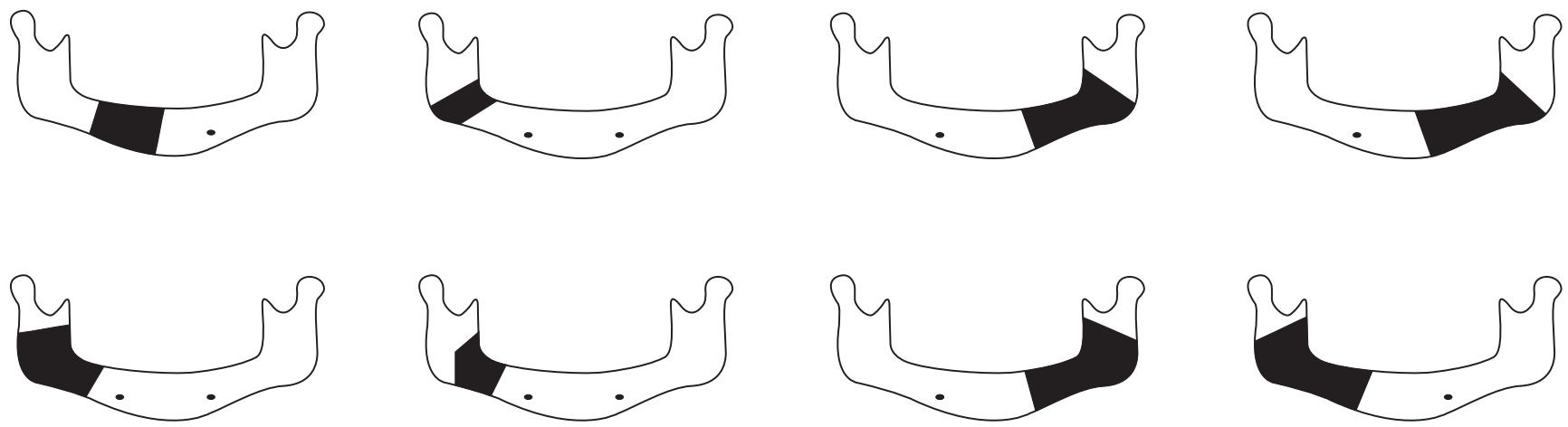

Fig. 6. Schematic images representing cases in which complications developed. A defect of the mandibular angle present in almost all cases. Guk-Jin Seol et al: Reconstruction plates used in the surgery for mandibular discontinuity defect. J Korean Assoc Oral Maxillofac Surg 2014 
struction plate screw loosening. Analysis of the hazard rates and overall survival rates (Fig. 5) indicated that plate fracture was the main cause of complications in the early stages following surgery (9.25 \pm 5.10 months), but screw loosening was the main complication in the later stages $(35.75 \pm 17.00$ months).

Most complications associated with the reconstruction plate developed in patients with a discontinuous defect in the mandibular angle area.(Fig. 6) These patients also had an anterior or contralateral dentulous condition. There was a statistically significant decrease in the survival rate of the reconstruction plates in patients who had received postoperative RT (log-rank test, $P=0.012$ ).(Fig. 7) The irradiated patients had a higher risk of plate-related complications. Of a total of 8 patients that received postoperative RT, 3 patients $(37.5 \%)$ experienced plate failures.

\section{Discussion}

The main goals of mandibular reconstruction are to achieve functional and esthetic recovery by restoring mandibular arch continuity, to maintain soft tissue coverage, and to improve the patient's postoperative quality of life. The success rate of mandibular reconstruction has increased as a result of advances in plate design and materials. However, plate-related complications still develop frequently and can sometimes cause serious problems for patients.

The plate fractures in this study occurred at approximately 10 months following surgery. Other studies have reported that most plate fatigue fractures occur within the first 6 months following surgery ${ }^{8-10}$. Currently, no studies have assessed coronoidectomy influence on the mandibular reconstruction plate. However, some studies have examined the mechanical forces that affect the plate. Kimura et al. ${ }^{11}$ found that masticatory pressure can contribute to vertical stress on the plate, leading to bone resorption around the screw and screw loosening. Arias-Gallo et al. ${ }^{12}$ reported that most hardware complications developed at sites exposed to higher moment and shear forces. Mandibular functional movements such as mastication cause mechanical stress, an important factor in complications. Forces that are caused by contracture of the masticatory muscles (temporalis muscle, lateral and medial pterygoid muscle, masseter muscle) during mastication act directly on the mandible ${ }^{13}$. The mandibular temporalis muscles are very powerful ${ }^{14}$ and are attached to the coronoid process of the mandible. As a consequence, when the surgical procedure includes a coronoidectomy, the force of the temporalis muscle is not delivered to the mandible, and so the stress on the reconstruction plate may be reduced, resulting in a decrease in plate-related complications. In this study, no mechanical complications were observed for any cases where the coronoid process had been removed.

Postoperative RT can be an important factor in plate-related complications and these are biological in origin. Previous authors have reported a decreased success rate following $\mathrm{RT}^{1,15,16}$. Other studies have reported that there is no relationship between success rate and $\mathrm{RT}^{17}$, which differs from the results of this study. Meanwhile, Maurer et al. ${ }^{1}$ reported that the first one-year success rate decreased according to the RT from $64 \%$ to $45 \%$, but this finding was not statistically significant $(P=0.67)$. The complications that occurred, according to RT, were infection, screw loosening and plate fracture.

RT damages small vessels, thus reducing the smooth muscle density and progressively thickens the sub-endothelial components of the vessel wall and leads to progressive occlusion and fibrosis of the vessels ${ }^{18,19}$. Marx ${ }^{20}$ has reported on the pathogenesis of jaw osteoradionecrosis which is characterized by a reduction of the number of vessels in the fibrotic periosteum, a reduction in the number of osteoblasts and osteocytes, and fibrosis of the marrow spaces. The resultant tissue hypovascularity and hypocellularity was exhibited hypoxic damage compared to non-irradiated tissue. A similar mechanism may be the cause of plate-related complications. In this study, the patient with RT experienced more mechanical complications of reconstruction plate, therefore additional study with a larger number of samples is needed.

The effectiveness of bone grafts with the reconstruction plate in surgery to correct mandibular discontinuous defects remains controversial. Some authors have reported that there is no statistical significance ${ }^{21}$, while others have found that the incidence of plate fracture was higher in patients that did not receive bone grafts ${ }^{22,23}$. In this study, there was a tendency towards a reduced risk of plate fracture in the patients who had received bone grafts ( 1 fracture out of 4 grafted cases), but this result was not statistically significant. There were no significant differences in plate survival rate according to age, gender, or defect size. These results were similar to conclusions of previous reports ${ }^{1,17}$.

Based on the results of this study, coronoidectomy is helpful for improving the plate survival rate in mandibular discontinuity defects, especially if the defect included the mandibular angle area. During the postoperative follow up period, it is better to carefully observe the complications such as plate fracture or screw loosening over time. Postoperative RT could increase the risk of reconstruction plate complications as well as other 
complications such as dysphagia and trismus. Therefore, it is important to prepare and educate patients and warn them of potential complications related reconstruction plate procedures.

\section{Conclusion}

The purpose of this study was to evaluate the factors associated with postoperative complications and analyze the mandibular reconstruction plate survival rate in mandibular discontinuity defect corrections. Differences in the survival rate were identified which depended on whether the patient underwent coronoidectomy and postoperative RT. The results indicated that the risk of the plate-related complications could be reduced by coronoidectomy and that postoperative RT has adverse effects on the plate survival rate. In contrast, age, gender, defect size, location of defect, and bone graft were not factors that affected plate survival rate in this study. In addition, the results indicated that the risk of plate fracture was relatively high in the 10 months following surgery. After 35 months, the risk of screw loosening became more significant.

Coronoidectomy should be considered in order to reduce mechanical complications that result from fitting a reconstruction plate, especially when the mandibular angle area is involved. RT can increase the likelihood of complications following mandibular reconstruction plate fitting. Therefore, surgeons need to make additional efforts to minimize surgical trauma in order to decrease postoperative complications, as well as provide explanations of potential complications to patients that will receive irradiation. Further studies would be useful to identify additional factors involved in the longterm survival rates of reconstruction plates and to confirm the effect of coronoidectomy to reduce reconstruction plate mechanical complications.

\section{Conflict of Interest}

No potential conflict of interest relevant to this article was reported.

\section{References}

1. Maurer P, Eckert AW, Kriwalsky MS, Schubert J. Scope and limitations of methods of mandibular reconstruction: a long-term followup. Br J Oral Maxillofac Surg 2010;48:100-4.

2. Schmoker R, Spiessl B, Mathys R. A total mandibular plate to bridge large defects of the mandible. In: Spiessl B, Bassetti C, eds. New concepts in maxillofacial bone surgery. Berlin, New York: Springer-Verlag; 1976:156-9.

3. Peacock ZS, Afshar S, Lukas SJ, Kaban LB. Customized repair of fractured mandibular reconstruction plates. J Oral Maxillofac Surg 2012;70:e563-73.

4. Knott PD, Suh JD, Nabili V, Sercarz JA, Head C, Abemayor E, et al. Evaluation of hardware-related complications in vascularized bone grafts with locking mandibular reconstruction plate fixation. Arch Otolaryngol Head Neck Surg 2007;133:1302-6.

5. Szypryt P, Forward D. The use and abuse of locking plates. Orthop Trauma 2009;23:281-90.

6. Klotch DW, Gal TJ, Gal RL. Assessment of plate use for mandibular reconstruction: has changing technology made a difference? Otolaryngol Head Neck Surg 1999;121:388-92.

7. Probst FA, Mast G, Ermer M, Gutwald R, Schmelzeisen R, Pautke $\mathrm{C}$, et al. MatrixMANDIBLE preformed reconstruction plates--a two-year two-institution experience in 71 patients. J Oral Maxillofac Surg 2012;70:e657-66.

8. Gellrich NC, Suarez-Cunqueiro MM, Otero-Cepeda XL, Schön R, Schmelzeisen R, Gutwald R. Comparative study of locking plates in mandibular reconstruction after ablative tumor surgery: THORP versus UniLOCK system. J Oral Maxillofac Surg 2004;62:186-93.

9. Katakura A, Shibahara T, Noma H, Yoshinari M. Material analysis of AO plate fracture cases. J Oral Maxillofac Surg 2004;62:348-52.

10. Lindqvist C, Söderholm AL, Salo A, Subasinghe J, Ylijoki S, Skutnabb K, et al. A comparative study on four screw-plate locking systems in sheep: a clinical and radiological study. Int J Oral Maxillofac Surg 2001;30:160-6.

11. Kimura A, Nagasao T, Kaneko T, Tamaki T, Miyamoto J, Nakajima T. Adaquate fixation of plates for stability during mandibular reconstruction. J Craniomaxillofac Surg 2006;34:193-200.

12. Arias-Gallo J, Maremonti P, González-Otero T, Gómez-García E, Burgueño-García M, Chamorro Pons M, et al. Long term results of reconstruction plates in lateral mandibular defects. Revision of nine cases. Auris Nasus Larynx 2004;31:57-63.

13. Nagasao T, Miyamoto J, Tamaki T, Kawana H. A comparison of stresses in implantation for grafted and plate-and-screw mandible reconstruction. Oral Surg Oral Med Oral Pathol Oral Radiol Endod 2010;109:346-56.

14. Faulkner MG, Hatcher DC, Hay A. A three-dimensional investigation of temporomandibular joint loading. J Biomech 1987;20:997-1002.

15. Schöning H, Emshoff R. Primary temporary AO plate reconstruction of the mandible. Oral Surg Oral Med Oral Pathol Oral Radiol Endod 1998;86:667-72.

16. Mariani PB, Kowalski LP, Magrin J. Reconstruction of large defects postmandibulectomy for oral cancer using plates and myocutaneous flaps: a long-term follow-up. Int J Oral Maxillofac Surg 2006;35:427-32.

17. Chepeha DB, Teknos TN, Fung K, Shargorodsky J, Sacco AG, Nussenbaum B, et al. Lateral oromandibular defect: when is it appropriate to use a bridging reconstruction plate combined with a soft tissue revascularized flap? Head Neck 2008;30:709-17.

18. Boyd TG, Huber KM, Verbist DE, Bumpous JM, Wilhelmi BJ. Case report removal of exposed titanium reconstruction plate after mandibular reconstruction with a free fibula osteocutaneous flap with large surgical pin cutters: a case report and literature review. Eplasty 2012;12:e42.

19. Olascoaga A, Vilar-Compte D, Poitevin-Chacón A, Contreras-Ruiz $\mathrm{J}$. Wound healing in radiated skin: pathophysiology and treatment options. Int Wound J 2008;5:246-57.

20. Marx RE. Osteoradionecrosis: a new concept of its pathophysiology. J Oral Maxillofac Surg 1983;41:283-8.

21. Head C, Alam D, Sercarz JA, Lee JT, Rawnsley JD, Berke GS, et al. Microvascular flap reconstruction of the mandible: a comparison of bone grafts and bridging plates for restoration of mandibular continuity. Otolaryngol Head Neck Surg 2003;129:48-54.

22. Shibahara T, Noma H, Furuya Y, Takaki R. Fracture of mandibular reconstruction plates used after tumor resection. J Oral Maxillofac Surg 2002;60:182-5.

23. Klotch DW, Prein J. Mandibular reconstruction using AO plates. Am J Surg 1987;154:384-8. 